\title{
Tiempo escolar e inclusión educativa: un estudio de teorías subjetivas de profesores
}

\author{
María P. Escobar, Dariam C. Muñoz, Camila D. Piñones y David J. Cuadra* \\ Departamento de Psicología, Facultad de Humanidades y Educación, Universidad de Atacama, Avenida Copayapu 485, \\ Copiapó, Chile (correo-e: david.cuadra@uda.cl; escobarcampusanomariapaz@gmail.com; \\ dariamcatalina95@gmail.com; camila.pinones.vega@gmail.com)
}

* Autor a quien debe ser dirigida la correspondencia.

Recibido Mar. 25, 2020; Aceptado May. 25, 2020; Versión final Jun. 30, 2020, Publicado Oct. 2020

\section{Resumen}

Este estudio buscó describir e interpretar las teorías subjetivas (TS) sobre el tiempo escolar y su relación con la inclusión educativa de profesores de tres regiones de Chile. Se utilizó un estudio descriptivo interpretativo, metodología cualitativa y un diseño de estudio de caso. El muestreo fue a priori y participaron 69 profesores y profesoras. Se aplicaron 47 entrevistas episódicas y 4 grupos de discusión. Los datos se analizaron en base a la teoría fundamentada y la técnica de análisis específico de TS. Se encontraron TS que relacionan el tiempo escolar con la inclusión educativa como un factor que principalmente dificulta esta tarea. Esto está asociado a un contenido emocional negativo de estas teorías subjetivas, que orienta la acción docente, limitando sus prácticas pedagógicas para la inclusión de los estudiantes. Se concluye que los profesores reconocen la relevancia del tiempo y la inclusión, en el contexto educativo, sin embargo, advierten de una limitada formación al respecto.

Palabras clave: tiempo escolar; inclusión educativa; formación docente; teorías subjetivas; estudiantado

\section{School time and educational inclusion: a study of subjective theories of teachers}

\begin{abstract}
This study sought to describe and interpret the subjective theories (STs) about school time and its relationship with the educational inclusion of teachers from three Chilean regions. This was an interpretive descriptive study with qualitative methodology and a case study design. The sample was a priori and 69 professors participated in the study. There were 47 episodic interviews and four discussion groups. Data were analyzed based on grounded theory and the technique of specific analysis of subjective theories. STs were found that relate school time to educational inclusion, a factor that mainly limited this task. This was associated with a negative emotional content of these subjective theories, which also guides the teaching action, limiting their pedagogical practices for the inclusion of students. It is concluded that teachers acknowledged the relevance of educational time and inclusion, but they were concerned about having limited training.
\end{abstract}

Keywords: school time; educational inclusion; teacher training; subjective theories; students 


\section{INTRODUCCIÓN}

Gran parte de las medidas de mejoramiento de la calidad educativa (Madani, 2019) se han basado en políticas de gestión y uso del tiempo escolar (Rubio et al., 2019). Por otra parte, la literatura científica también le asigna al tiempo escolar un rol importante en la comprensión de los fenómenos educativos, como la enseñanza, el aprendizaje y la formación docente, entre otros (Adams \& Blair, 2019). En el caso del profesorado, el tiempo escolar cobra importancia, porque el significado que le otorga al tiempo, su uso y gestión influye en el destino de las futuras generaciones. Una parte importante del desempeño docente se encuentra relacionado con el uso de este recurso, teniendo una incidencia significativa en la eficacia educativa (Ather et al., 2016). Por ejemplo, hay evidencia de que la sobre carga de trabajo tiene un impacto negativo en el estrés docente, como así también en el rendimiento académico de los estudiantes (Berda \& Ochieng, 2019). Esta problemática se acentúa en los países no desarrollados o en vías de desarrollo y sobre todo en Latinoamérica, por la cantidad de funciones y tareas que deben desarrollar los profesores (Rubio et al., 2019), además de las cada vez mayores demandas sociales por educar bajo principios y objetivos que aseguren adecuados desempeños en el mundo laboral, un comportamiento ciudadano ético y competencias para enfrentar los desafíos socio ambientales que tensionan la subsistencia del ecosistema.

La inclusión educativa es justamente una demanda social y un derecho de la ciudadanía, por lo tanto, un deber de las escuelas. La educación inclusiva es el enfoque educativo mediante el cual todos los niños y niñas aprenden juntos, independientemente de su raza, cultura, género, religión, necesidades educativas especiales y nivel socioeconómico. Sin embargo, implica un desafío tanto para países desarrollados como no desarrollados, siendo algunos de los problemas importantes para hacerla efectiva, la sobre carga de trabajo docente y las dificultades en la gestión y uso del tiempo de las escuelas (Massouti, 2019).

Una línea importante de investigación ha abordado las creencias que los profesores tienen acerca de la inclusión educativa. Básicamente, en estos trabajos se ha encontrado que los significados docentes sobre la inclusión inciden en las prácticas pedagógicas inclusivas, limitándolas o favoreciéndolas (Sharma \& Sokal, 2015). En estas investigaciones, una creencia frecuente del profesorado es el factor tiempo como obstáculo para la inclusión educativa (Massouti, 2019), siendo la reflexión sobre sus propias creencias y la oportunidad de generar experiencias exitosas de inclusión educativa, un paso necesario para lograr mejores desempeños docentes al respecto.

En este trabajo se busca comprender qué significado le otorgan los profesores al tiempo escolar y cómo lo relacionan con la inclusión educativa. Se aborda el conocimiento docente desde las teorías subjetivas (en adelante TS), definidas como un tipo de creencia con estructura argumentativa, similar a las teorías científicas por su capacidad de explicar, predecir, justificar y orientar el comportamiento humano (Flick, 2019), además de tener un fuerte arraigo en la estructura cognitiva, a menudo parcialmente implícito (Przibilla et al., 2018), condicionando el aprendizaje de teorías formales o científicas. Las TS se enmarcan en un paradigma humanista o fenomenológico, dado que desde este programa de investigación, se asume al sujeto como un constructor de realidades y al conocimiento, como una construcción cognitiva y social (Catalán, 2016). En educación, hay una vasta literatura de TS de profesores, que señala la implicancia que este tipo de creencia tiene para el profesorado, en la comprensión de las problemáticas educativas y la toma de decisiones profesionales.

En 1994, la National Education Commission on Time and Learning, de los Estados Unidos, publicó un reporte sobre los principales desafíos que las escuelas deben asumir para utilizar más efectivamente el tiempo. Una década después, en un nuevo reporte, esta misma comisión advirtió de la invariabilidad en el uso del tiempo escolar para el aprendizaje y la dificultad de generar cambios al respecto. Actualmente, este equipo de trabajo alerta de la necesidad de lograr una mayor comprensión científica de cómo las escuelas usan el tiempo, para producir mejoras educativas (Dougherty et al., 2019).

El tiempo escolar ha sido conceptualizado como un símbolo de democratización, una oportunidad de aprendizaje, un recurso para la calidad educativa, e incluso, como un catalizador para la innovación educativa y pedagógica (Garzia et al., 2016; Wang, 2011). En contexto educativo, se considera como un recurso importante y básico que deben saber gestionar los profesionales del siglo XXI (Álvarez \& Lobo, 2020). A menudo, el tiempo escolar es definido en base a su dimensión objetiva o subjetiva. La primera de éstas, el "tiempo en la escuela", entiende el tiempo como monocrónico, externo, instrumental, ordenado y secuenciado (Rubio et al., 2019). Un número importante de investigaciones ha estudiado el tiempo escolar desde este enfoque, con la meta de determinar cuantitativamente el efecto del incremento o disminución del tiempo escolar en los resultados en términos de aprendizaje (Peng et al., 2019; Wang, 2011). Por otro lado, el tiempo escolar subjetivo o "tiempo de la escuela", destaca el sentido y significado que actores educativos le asignan a este recurso. Implica una construcción social, con base en la cultura y una duración que incluso no se intersecta con el tiempo objetivo. Este tipo de investigaciones son menores respecto de aquellas que 
se han focalizado en el tiempo objetivo (Rubio et al., 2019). El tiempo escolar también es entendido en base a los períodos relacionados con los procesos educativos. Algunos teóricos proponen distinguir el tiempo dentro del horario escolar, del tiempo fuera del horario escolar; el tiempo de enseñanza (destinado a enseñar una lección) del tiempo de aprendizaje (el que transcurre cuando realmente los estudiantes se involucran con la enseñanza); el tiempo de procedimiento, que consiste en el período requerido para mantener el orden en el aula (Garzia et al., 2016), entre otras categorías más específicas aún.

\section{OTROS ANTECEDENTES}

Desde una perspectiva subjetiva y en base a cómo los profesores le otorgan significado a este recurso, se ha encontrado que el tiempo es un factor omnipresente en la subjetividad del profesorado, siendo considerado como un obstáculo o facilitador del desarrollo de los estudiantes (Ather et al., 2016), de su propio desempeño e incluso de su desarrollo personal (Demirkasimoglu, 2015). Aunque los estudios internacionales se focalizan en realidades culturales distintas a la chilena, dan luces sobre cómo el tiempo es interpretado por el profesorado. Wang (2011) encontró que profesores rurales chinos consideran el recurso tiempo como un factor que impide implementar estrategias de enseñanza centradas en el alumno, porque este último enfoque requiere de una alta proporción de tiempo. En profesores suecos se encontró un resultado similar al anterior: la escasez de tiempo limita la posibilidad de reflexionar el tipo de retroalimentación que un maestro proporciona al estudiante mientras aprende, y también impide promover la reflexión del estudiante durante esta dinámica (Eriksson et al., 2018). En profesores chilenos hay evidencia de que elaboran TS del tiempo que se caracterizan por un significado emocional negativo, que implica emociones como la ansiedad e inseguridad, además de explicaciones externas e incontrolables que generan desafíos para la gestión del tiempo, al limitar la agencia del maestro (Castro et al., 2019). Otro resultado relevante y altamente frecuente es la consideración de una escasez de tiempo, asociado a las altas demandas laborales y a consecuencias adversas de esta escasez, que afectan negativamente la calidad de la enseñanza y la vida personal, por ejemplo, en profesores turkos (Demirkasimoglu, 2015). También se ha encontrado que las innovaciones pedagógicas incluyen el factor tiempo como una variable clave en la viabilidad del cambio, inhibiendo o promoviendo la adherencia del profesorado a la implementación de las nuevas prácticas pedagógicas (Esteban-Guitar et al., 2019).

La educación inclusiva está orientada a que todos los niños y niñas aprendan juntos, independientemente de su raza, cultura, género, religión, necesidades educativas especiales y nivel socioeconómico. En América Latina, este concepto aparece asociado al de democratización. Básicamente, una escuela democrática es aquella que genera las condiciones para que todos los estudiantes avancen hacia el éxito educativo y democratiza las aulas para incluir activamente a todos los actores educativos, en la toma de decisiones de la comunidad escolar (Moliner et al., 2016). Para Amor et al. (2019), un establecimiento educativo es inclusivo cuando todos los estudiantes están presentes, participan, aprenden y reciben instrucción con sus pares, durante todo o parte de un día escolar. Booth y Ainscow (2011), han elaborado un Índice de Inclusión para las escuelas, que representa un marco para la planificación de una enseñanza inclusiva. Así, desde estos autores, se sugiere que para implementar una educación inclusiva, es necesario que primeramente las escuelas conozcan y comprendan sus propios desafíos, recursos, valores y cultura. Aunque las políticas de educación inclusiva son de larga data, el progreso logrado al respecto ha sido muy lento, lo que alerta de la necesidad de estudiar mayormente el problema (Hagiwara et al., 2019). Un problema al respecto se refiere a los significados y actitudes de los profesores hacia la inclusión (Sharma \& Sokal, 2015). En el trabajo de Pick et al., (2016), una de las conclusiones más importantes es que se debe fortalecer la actitud inclusiva y la reflexión de los docentes, para mejorar la inclusión de los estudiantes. Al contrario del trabajo de Pick et al. (2016), Aas (2019) encontró en profesores noruegos una actitud favorable para la inclusión educativa, pero creencias que limitan este proceso, dado que consideran que las necesidades educativas especiales son un problema individual del estudiante y la adaptación un esfuerzo personal del mismo, por lo que el docente tendría un limitado rol al respecto. En profesionales alemanes se ha encontrado resultados similares, puesto que utilizan predominantemente teorías subjetivas o intuitivas para la toma de decisiones profesionales sobre la inclusión que distan de manera importante del saber formal (Knauf \& Graffe, 2016).

Un hallazgo frecuente en las investigaciones sobre creencias docentes acerca de la educación inclusiva, es que el tiempo se constituye como un factor obstaculizador o facilitador (Massouti, 2019). Básicamente, los maestros argumentan que la insuficiencia del recurso tiempo o la inadecuada gestión del mismo es una de las causas de la dificultad para instalar prácticas pedagógicas inclusivas, por lo que se requiere de más tiempo para abordar las necesidades de los estudiantes. Considerando lo anterior, es que este trabajo busca describir e interpretar las teorías subjetivas (TS) sobre el tiempo escolar y su relación con la inclusión educativa, de profesores de instituciones educativas de tres regiones de Chile. A nivel específico se busca: (a) identificar y describir el significado de tiempo escolar e inclusión educativa y la relación entre estos conceptos; (b) determinar factores que promueven y obstaculizan la inclusión educativa, en función del tiempo escolar; (c) describir la forma en que los profesores construyen las TS sobre el tiempo escolar e 
inclusión educativa; (d) describir medidas o propuestas de estos docentes para la inclusión educativa, en función del tiempo escolar y (e) proponer un modelo comprensivo acerca de cómo el tiempo escolar se relaciona con la inclusión educativa y las prácticas pedagógicas.

\section{MÉTODO}

Corresponde a un estudio descriptivo interpretativo, que utiliza metodología cualitativa. En este tipo de estudios, se busca abordar los problemas de investigación desde el conocimiento cotidiano o subjetivo (Taylor \& Bogdan, 1987). El diseño es de estudio caso, definido como aquel que busca describir en profundidad las características de un caso que por su particularidad es de interés para el estudio (Flick, 2019). En este trabajo, se consideró como caso a profesores que se desempeñan en los niveles educativos más representativos de la educación chilena: educación especial, educación preescolar, educación básica y educación secundaria, omitiendo a quienes imparten clases en educación superior. El muestreo es intencionado y por conveniencia (Flick, 2019) y corresponde a profesores y profesoras de tres regiones de Chile (Atacama, Coquimbo y Concepción), tomando como criterios de representatividad cualitativa de caso el género, los años de experiencia, el grupo socioeconómico (NSE) del establecimiento y el nivel en el que enseñan. Como criterios de inclusión se consideró poseer el título profesional de profesor y tener al menos 1 año de experiencia en el ejercicio de la docencia. Las condiciones anteriores se utilizaron para seleccionar a los profesores y profesoras de este estudio. La tabla 1 muestra detalladamente las características del muestreo, en función de los criterios de representatividad cualitativa de caso. En esta tabla se abrevia grupo de discusión como GD; mujer como M y hombre como H. Además, algunos profesores participaron de ambos instrumentos de recolección de información.

Tabla 1: Características del muestreo

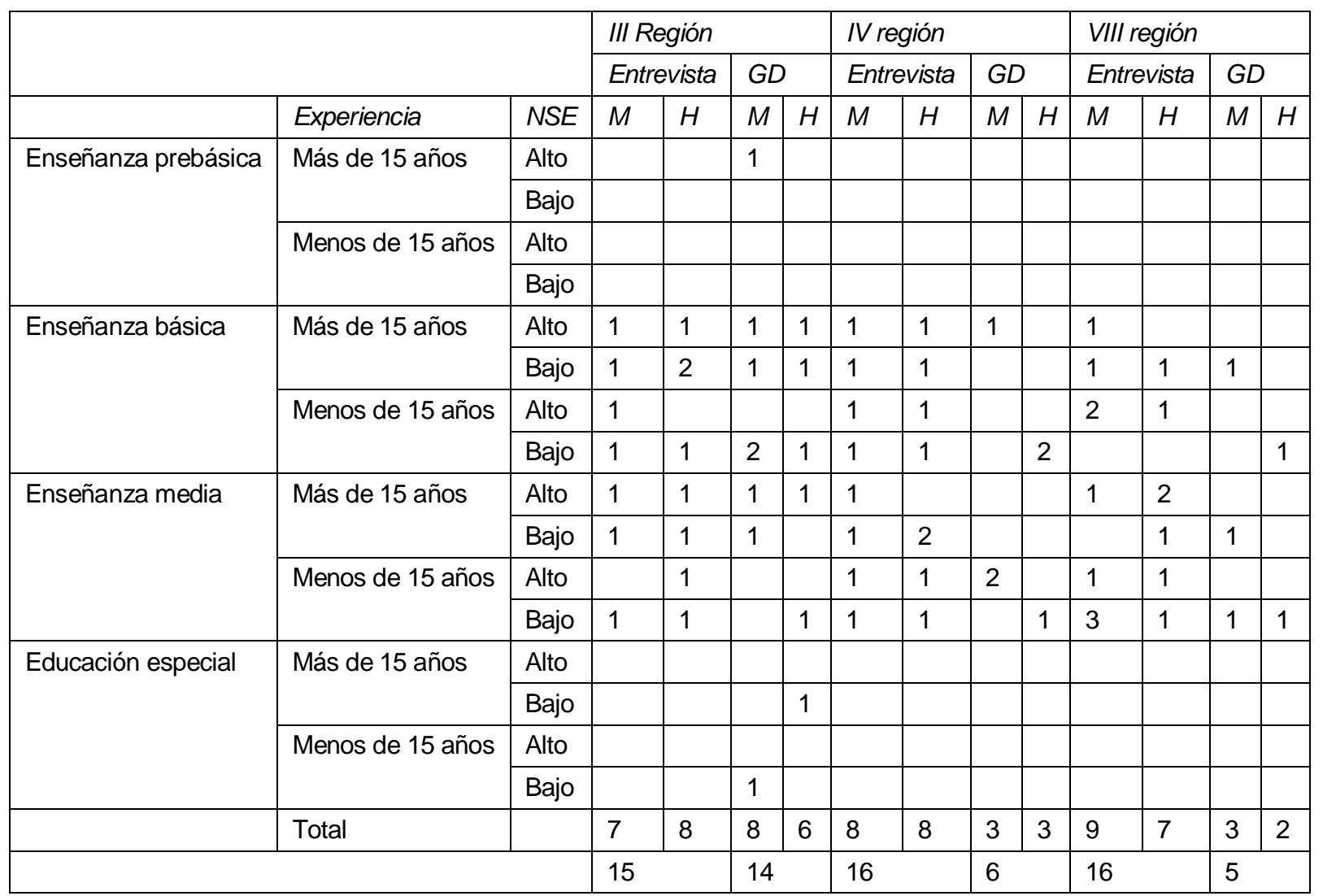

\section{Procedimiento de recolección de información}

Este trabajo contó con la aprobación del comité ético y científico de una universidad del norte de Chile. Se contactó a los profesores en cada una de las regiones y se les explicó los fines del estudio y los criterios éticos. Se procedió a solicitarles la firma de un consentimiento informado para posteriormente proceder a aplicar los instrumentos. Entrevista episódica: Esta entrevista es uno de los instrumentos recomendado para la reconstrucción de teorías subjetivas individuales, porque facilita su reconstrucción a partir de las experiencias biográficas de los participantes. Procedimentalmente, se solicitó a los profesores recordar experiencias profesionales y elaborar explicaciones sobre éstas, realizar definiciones de conceptos, además de elaborar explicaciones en base a episodios hipotéticos propuestos por el investigador. Se realizaron 47 entrevistas 
episódicas en los establecimientos educativos (Flick, 2019), bajo la siguiente guía temática. (a) significados de tiempo escolar, (b) relación entre el tiempo escolar e inclusión educativa; (c) obstaculizadores y facilitadores de la inclusión educativa, en función del tiempo y (d) sugerencias para la inclusión educativa, en función del tiempo escolar. Los datos se grabaron en audio y aproximadamente tuvieron una duración de 1 hora por entrevista.

Grupo de discusión: Se realizaron 4 grupos de discusión, uno en la IV región, uno en la VIII y dos en la III región. Se conformaron grupos con un tamaño de 5 a 8 participantes. Para conformar los grupos de discusión se buscó alcanzar la máxima heterogeneidad de acuerdo a los criterios de diferenciación de la muestra. La estructura temática de la entrevista episódica fue reformulada a partir del análisis de datos proveniente de las entrevistas, lo que permitió orientar este segundo proceso de indagación con la finalidad de contrastar y profundizar los resultados preliminares, opción metodológica que también aportó mayor credibilidad al estudio, en la medida que permitió una triangulación de instrumentos de recolección de información. El guión temático consideró: (a) una breve presentación oral de los modelos de codificación axial y selectiva y (b) observaciones de los participantes sobre estos modelos. Al igual que para las entrevistas, se centró en la indagación de narraciones de situaciones o experiencias en relación con el tiempo escolar y la propuesta de Flick (2019) para la entrevista episódica.

\section{Procedimiento de análisis de datos}

Para analizar los datos se utilizó la técnica de análisis de la teoría fundamentada, que tiene el fin de crear categorías iniciales e ir descubriendo relaciones entre los conceptos a través de las distintas codificaciones (abierta, axial y selectiva) (Strauss \& Corbin, 1998), las cuales se hicieron en conjunto por los investigadores a través del software ATLAS. Ti 7. En la codificación abierta, se identificaron los conceptos y se descubrieron en los datos sus propiedades y dimensiones. El fin fue hacer conceptualizaciones, definir categorías y desarrollarlas en término a sus propiedades y dimensiones para luego relacionarlas. En la codificación axial, se relacionaron las categorías a sus subcategorías siguiendo las líneas de sus propiedades y dimensiones y de mirar cómo se entrecruzan y vinculan éstas. Se agruparon los datos que se encontraron durante la codificación abierta, es decir, las categorías se relacionaron con sus subcategorías formando explicaciones más precisas y completas sobre los sujetos. En la codificación selectiva, se integraron y refinaron la teoría, se redujeron datos de muchos casos a conceptos y se convirtieron conjuntos de afirmaciones de relación que se utilizaron para explicar lo que ocurre (Strauss \& Corbin, 1998).

\section{RESULTADOS Y DISCUSIÓN}

En primer lugar se expone la codificación abierta. Posteriormente, para cada categoría semántica se presenta la codificación axial, para finalmente graficar la codificación selectiva. Las codificaciones axial y selectiva se describen siguiendo la sugerencia de Flick (2019), presentando un mapa de conceptos y un breve párrafo explicativo.

\section{Codificación abierta: Tiempo, inclusión y formación docente}

Para los docentes, la inclusión educativa y el tiempo escolar son factores importantes de profesionalizar para ejercer el rol de educador. De esta manera, se presenta una posición ampliamente compartida respecto de la necesidad de tener formación en la temática. Así lo expresa un profesor (Hombre, NSE bajo, Enseñanza media, menos de 15 años): "Respecto al tema de tiempo escolar, ahí yo me di cuenta de lo importante que es la formación de la inclusión en la formación de pregrado de todas las carreras, porque si no pasa esto que de repente hay conceptos que todavía la gente no maneja y la inclusión no va a avanzar". Se observan dos subcategorías que se presentan en la tabla 2, que incluyen TS de contenido emocional positivo y negativo y que explican la forma en que los profesores aprenden acerca del tiempo escolar y la inclusión educativa.

Tabla 2: Teorías subjetivas acerca del tiempo escolar, la inclusión educativa y la formación docente

\begin{tabular}{|l|l|l|l|}
\hline TS supraordenada & TS encontradas & Contenido emocional & $\begin{array}{l}\text { Orientación } \\
\text { a la acción }\end{array}$ \\
\hline $\begin{array}{l}\text { TS sobre la } \\
\text { formación inicial }\end{array}$ & $\begin{array}{l}\text { La formación inicial es un espacio } \\
\text { importante, porque en ésta se podría } \\
\text { aprender de inclusión y tiempo escolar }\end{array}$ & $\begin{array}{l}\text { Ts de contenido emocional } \\
\text { positivo, hacia la formación } \\
\text { superior en tiempo escolar e } \\
\text { inclusión: valora este } \\
\text { espacio formativo }\end{array}$ & $\begin{array}{l}\text { Promueve } \\
\text { este tipo de } \\
\text { formación }\end{array}$ \\
\cline { 2 - 4 } & $\begin{array}{l}\text { La formación inicial en tiempo escolar e } \\
\text { inclusión educativa es inadecuada, porque } \\
\text { los programas educativos no se ajustan ni } \\
\text { al tiempo actual ni a la realidad escolar. }\end{array}$ & $\begin{array}{l}\text { Ts de contiva: desvalora este } \\
\text { negpacio formativo }\end{array}$ & $\begin{array}{l}\text { Inhibe este } \\
\text { tipo de } \\
\text { formación } \\
\text { profesional }\end{array}$ \\
\hline
\end{tabular}


Tabla 2: continuación.

\begin{tabular}{|l|l|l|l|}
\hline TS supraordenada & TS encontradas & Contenido emocional & $\begin{array}{l}\text { Orientación } \\
\text { a la acción }\end{array}$ \\
\hline $\begin{array}{l}\text { TS sobre la } \\
\text { formación a través } \\
\text { de la experiencia } \\
\text { profesional }\end{array}$ & $\begin{array}{l}\text { La experiencia profesional es un espacio } \\
\text { importante para el aprendizaje sobre } \\
\text { tiempo escolar e inclusión, dado que la } \\
\text { formación inicial actual no aborda } \\
\text { adecuadamente estos conceptos. }\end{array}$ & $\begin{array}{l}\text { Ts de contenido emocional } \\
\text { positivo: valora este espacio } \\
\text { formativo }\end{array}$ & $\begin{array}{l}\text { Promueve } \\
\text { este tipo de } \\
\text { formación }\end{array}$ \\
\cline { 2 - 4 } & $\begin{array}{l}\text { El aprendizaje sobre tiempo escolar e } \\
\text { inclusión durante la experiencia profesional } \\
\text { es inadecuado, si la institución educativa } \\
\text { presenta malas prácticas sobre el tema. }\end{array}$ & $\begin{array}{l}\text { Ts de contenido emocional } \\
\text { negativo: inseguridad y } \\
\text { suspicacia frente a este tipo } \\
\text { de experiencias formativas }\end{array}$ & $\begin{array}{l}\text { Inhibe este } \\
\text { tipo de } \\
\text { formación }\end{array}$ \\
\hline
\end{tabular}

Finalmente, también se explica que implementar prácticas pedagógicas inclusivas es difícil, dada las características de la formación inicial y el contexto de ejercicio profesional actual que se encuentra permeado por la escasez de tiempo. La carencia de tiempo se asocia con (a) factores actitudinales del docente que disminuyen la motivación y compromiso por la inclusión educativa, (b) factores cognitivos del docente, como una baja capacidad y motivación por gestionar el tiempo y aplicar adecuaciones curriculares; (c) factores estructurales, como políticas y micropolíticas de estandarización de la enseñanza que cuantifican el tiempo; (d) factores económicos referidos a una carencia de recursos financieros para favorecer la inclusión educativa. Lo anterior, repercute en prácticas pedagógicas que tienden a no respetar la diversidad, a evitar la adecuación de la enseñanza y a no favorecer suficientemente la inclusión de los estudiantes en los espacios escolares de socialización, en definitiva, a una estandarización de la enseñanza. Hombre, Enseñanza media, menos de 15 años, NSE bajo: En la mala experiencia de la práctica, (...) cuando uno entra a trabajar, piensa en todas estas actividades maravillosas que uno quiere hacer para los niños. Yo entre a escuela, el primer día me dormí a las 1 de la mañana haciendo material para el día siguiente, entonces en las malas experiencias de agobio uno se da cuenta de la importancia de los dos tiempos en escolares, del lectivo y del no lectivo y sobre inclusión.

\section{Codificación axial: Tiempo, inclusión y formación docente}

El conocimiento sobre el tiempo escolar y la inclusión educativa se adquiere a partir de la formación inicial y el ejercicio profesional, bajo ciertas condiciones. Cuando los programas de estudio de la formación inicial son actualizados y coherentes con las necesidades y problemáticas actuales, favorecen una adecuada formación profesional en la gestión del tiempo escolar para la inclusión educativa. Esto se fortalece mediante el ejercicio profesional, siempre que la comunidad educativa modele adecuadamente el uso del tiempo para la inclusión educativa. Cuando ocurre lo contrario, las prácticas pedagógicas tienden a un abordaje inadecuado de la inclusión educativa (Figura 1).

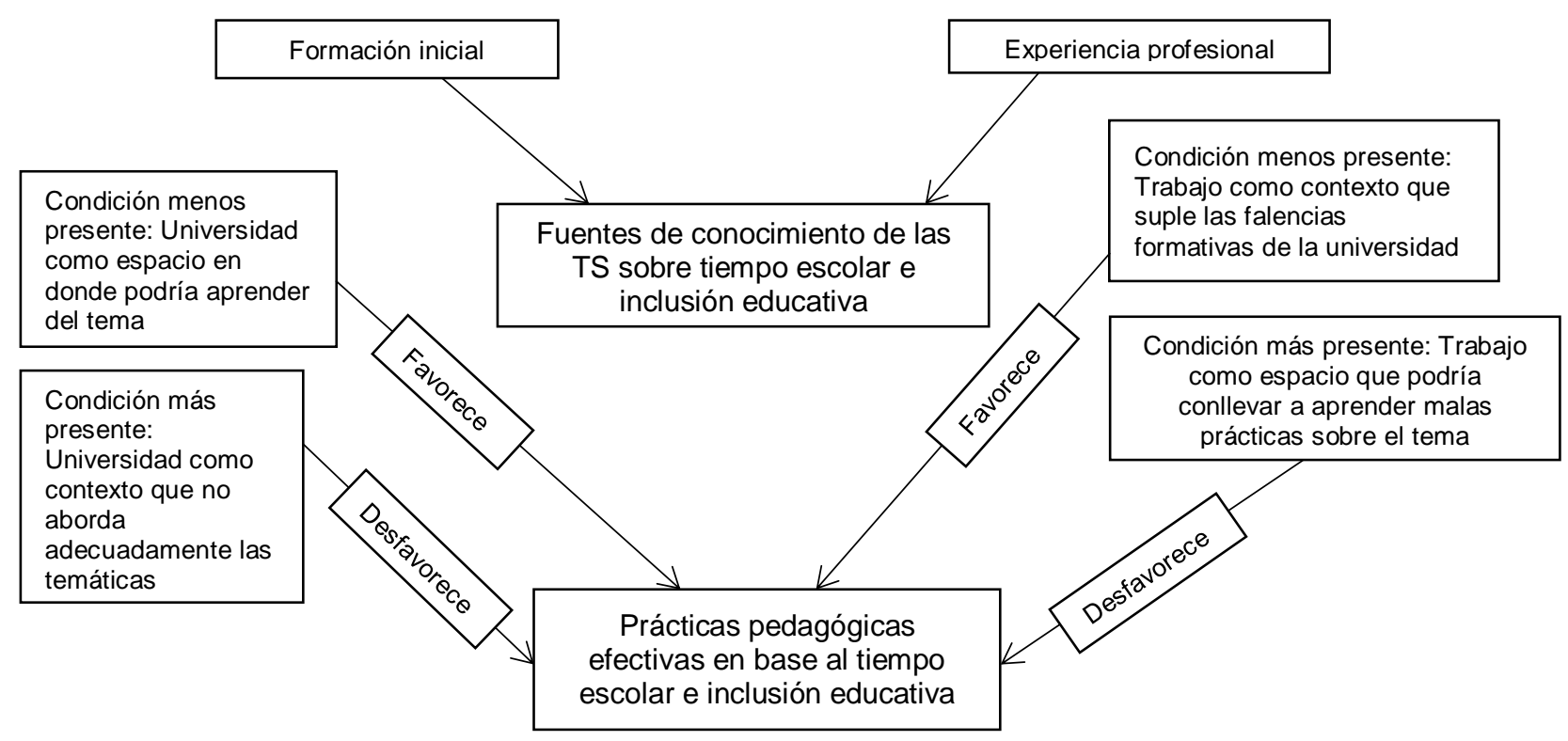

Fig. 1: Codificación axial tiempo, inclusión y formación docente. 


\section{Codificación abierta. Tiempo no lectivo: planificación de la inclusión}

Se observan tres TS que sustentan el ejercicio profesional docente, de planificación de la enseñanza para la inclusión de los estudiantes. La primera TS considera a las capacidades profesionales y organizacionales, como factores que determinan la planificación para la inclusión educativa. Los profesores explican que no poseen las competencias suficientes para planificar adecuadamente la enseñanza para la diversidad estudiantil. Dentro de esto, una de las principales limitaciones se refiere a la capacidad para planificar el tiempo de enseñanza, para una cada vez mayor población de estudiantes diversos. En segundo lugar, también se indica que la organización escolar no tiene la capacidad para otorgar una carga académica adecuada y el tiempo suficiente para planificar la enseñanza, considerando la diversidad estudiantil. Mujer, Enseñanza media, NSE bajo, menos de 15 años: Somos evaluados externamente por pruebas de medición, entonces eso hace que los colegas caigan en la monotonía justamente de las bases curriculares, de lo que tienen que hacer con los niños,(...) entonces muy poco se aprovecha el tiempo por algo realmente productivo pensando en las diferencias que hay dentro de un curso. Una segunda TS asocia la diversidad estudiantil, con un mayor consumo de tiempo escolar para planificar la enseñanza. Esto, bajo los siguientes argumentos: (a) la enseñanza se instala desde el conocimiento acerca de cómo un estudiante aprende, por lo que mientras más diversidad estudiantil hay, más difícil es conocer al estudiante y planificar la enseñanza, conllevando a que el docente tienda a utilizar planificaciones estándares; (b) todos los estudiantes son diversos, porque tienen distintas experiencias, personalidades, necesidades y ritmos de aprendizaje. Ambos argumentos alertan al docente de una necesidad de planificar la enseñanza en base a la asignación de más tiempo para apoyar a estos estudiantes. Mujer, menos de 15 años, Enseñanza básica, NSE bajo: ... sin ninguna cabida para reflexionar frente a lo que voy hacer con cada curso que es diverso y cómo voy a cambiar yo esa planificación de acuerdo al curso y a las personas que me enfrento, si los estudiantes son personas diversas, entonces ahí creo que se conjugan dos tiempos mal diseñados y mal enfocados. Finalmente, una tercera TS corresponde a una asunción ontológica de la educación, definiéndola como un proceso imprevisible, por lo que se debe actuar desde la emergencia y reactividad, situaciones que no son posibles de planificar y consumen el recurso temporal. Hombre, más de15 años, Enseñanza básica, NSE bajo: ... muchas veces te pasa que surgió una dificultad en la actividad que se estaba realizando y que no estaba contemplada... por algún problema que hizo que ellos se retrasaran en el avance de la actividad y ocuparon un poco más de tiempo, entonces todo depende dentro del aula del ritmo de trabajo de los alumnos. Estas tres TS se caracterizan por un contenido emocional de desesperanza y podrían orientar la acción docente inhibiendo las prácticas pedagógicas de planificación de la inclusión educativa y del tiempo para la inclusión.

\section{Codificación axial: tiempo escolar, planificación e inclusión educativa}

Se presentan tres TS supraordenadas, con argumentos ontológicos acerca de la educación y la diversidad, considerando a lo educativo como un proceso imprevisible y a la diversidad como una condición del estudiantado, situación que podría conllevar a que el tiempo escolar se considere como insuficiente para lograr una adecuada planificación de la enseñanza inclusiva. Esto genera desesperanza e inhibe las acciones docentes orientadas a planificar la inclusión y el tiempo para la inclusión (Figura 2).

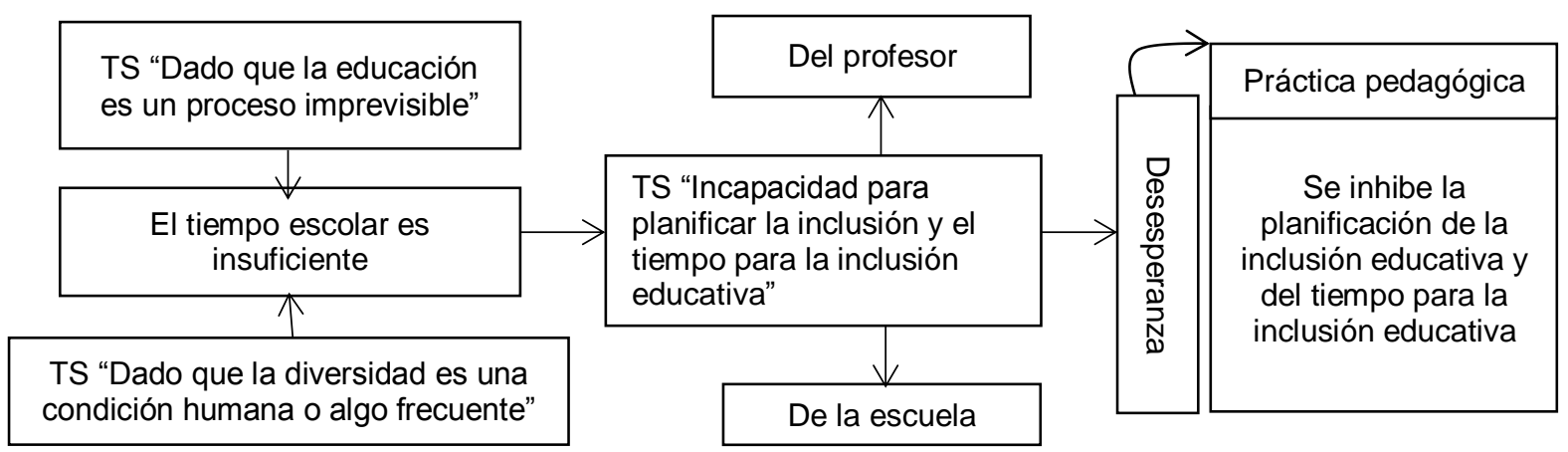

Fig. 2: Codificación axial tiempo escolar, planificación e inclusión educativa

\section{Obstaculizadores y facilitadores del uso del tiempo}

Dentro de los obstaculizadores, se identifican como causas factores políticos, sociales, psicológicos, técnicos y de experticia docente. Mientras que como facilitadores, se nombra la gestión del tiempo, la gestión de recursos y una filosofía inclusiva. Así lo expresan algunos participantes: (Mujer, NSE bajo, 
Enseñanza media, menos de 15 años) "La legislación del año pasado es la misma de hace 10 años atrás, entonces hay monotonía, porque no tienen tiempo... desde ese punto de vista las planificaciones son monótonas y no hay cambio ni con la ley de inclusión ni lo que uno quiera imponer en ese sentido"; (Mujer, NSE bajo, Enseñanza media, menos de 15 años) "Yo creo que va por el UTP y los directivos, con la visión que tienen ellos porque en el caso mío ellos tienen la visión inclusiva"; (Mujer, menos de 15 años, Enseñanza media, NSE alto).

\section{Codificación axial. Obstaculizadores y facilitadores del tiempo escolar}

Se presentan condiciones externas e internas a la escuela que pueden actuar como facilitadores $u$ obstaculizadores de la gestión del tiempo para la inclusión educativa. Si bien las condiciones internas de la escuela son sentidas con un mayor control docente, actúan tanto como facilitadores y obstaculizadoras del uso de este recurso. Mientras que las condiciones externas, sentidas como incontrolables y obstaculizadoras, predominan en este modelo, por lo que finalmente se produce una importante dificultad para lograr la inclusión educativa, en función del recurso tiempo (Figura 3).

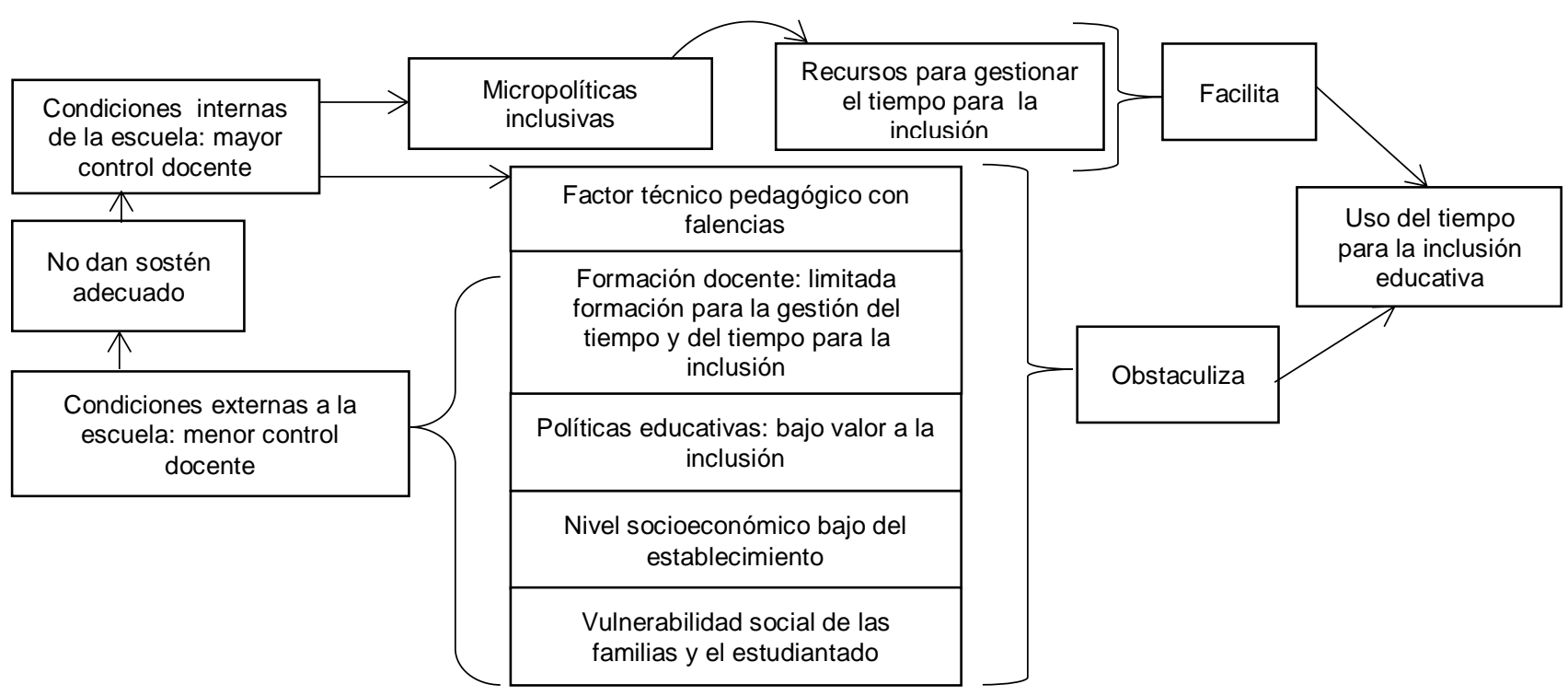

Fig. 3: Codificación axial obstaculizadores y facilitadores de la gestión del tiempo para la inclusión educativa

\section{Codificación abierta. Sugerencias del profesorado para el uso del tiempo}

La primera sugerencia teoriza que el tiempo escolar debería ser utilizado más eficientemente por el profesorado y la escuela, eficiencia que conllevaría a destinar el tiempo necesario para favorecer una educación inclusiva. En el caso del profesorado, el aprovechamiento del tiempo escolar se refiere a una mayor focalización docente en aspectos educativos y a una mejor gestión de este recurso. Esta TS podría orientar la acción hacia el adecuado uso del tiempo, incluyendo además un locus de control interno, lo que se podría asociar con la agencia docente. Mujer, más de 15 años, Enseñanza básica, NSE bajo: ese colega que no ocupa el tiempo, (...) tiene más dificultad para sacar adelante a su curso , que llega más a improvisar (...), entonces ahí ese colega que no ocupa el tiempo, no lo optimiza, y el colega que sí lo ocupa hace una buena labor, trabaja en el tiempo... La segunda TS destaca dos argumentos encadenados que podrían facilitar la gestión del tiempo para la inclusión educativa. El primer argumento se sustenta en la calidad de la formación profesional de los asistentes de la educación que trabajan en educación inclusiva: una mejor formación profesional de estos equipos, podría hacer más eficiente la inclusión de los estudiantes; lo contrario, dificulta y sobrecarga la función docente. El segundo argumento, es que lo anterior se resolvería al dotar de mayores recurso financieros a los establecimientos educativos, para la contratación de profesionales de apoyo con mejor calidad profesional. Mujer, más de15 años, Enseñanza básica, NSE bajo: necesitas a un profesional que acompañe al niño durante la jornada y se cumple siempre con el mínimo, no se invierte monetariamente.

\section{Codificación axial: Sugerencias del profesorado}

La TS identifica factores personales del profesor y factores escolares, que podrían facilitar el uso del tiempo para la inclusión educativa. Los factores personales del profesor implican un mayor grado de control sobre el uso y gestión del tiempo escolar para la inclusión, en cambio, los factores escolares consideran un sentimiento de menor control, determinando así una mayor o menor agencia docente en el tema (Figura 4). 


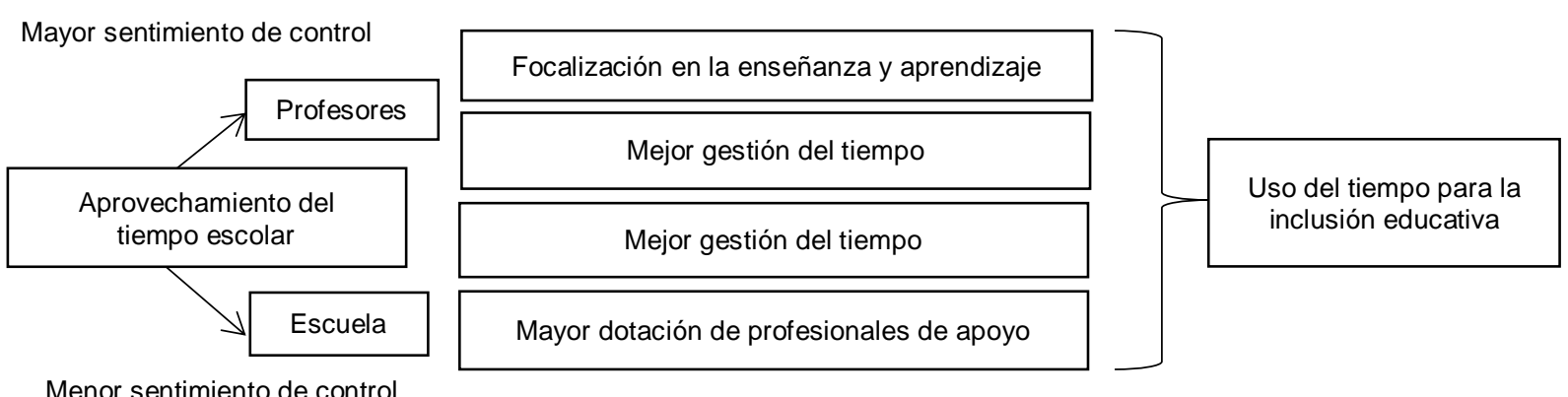

Fig. 4: Codificación axial. Sugerencias del profesorado para el uso del tiempo en la inclusión

\section{Codificación selectiva. Modelo comprensivo de TS}

El uso y gestión del tiempo para la inclusión educativa se sustenta en una TS supraordenada y central que relaciona TS sobre asunciones ontológicas de lo que es educar, significados del tiempo y la inclusión educativa, caracterizaciones del contexto educativo y del ser profesor, argumentos interconectados que explican la dificultad para la inclusión educativa en base al tiempo escolar. Estos argumentos se encadenan y se asocian a un contenido emocional ansioso, un bajo sentido de autoeficacia, malestar y agobio, orientando la acción docente con un sobre esfuerzo y agotamiento para el uso del tiempo en la inclusión de los estudiantes o al descuido y abandono de la tarea inclusiva (Figura 5).

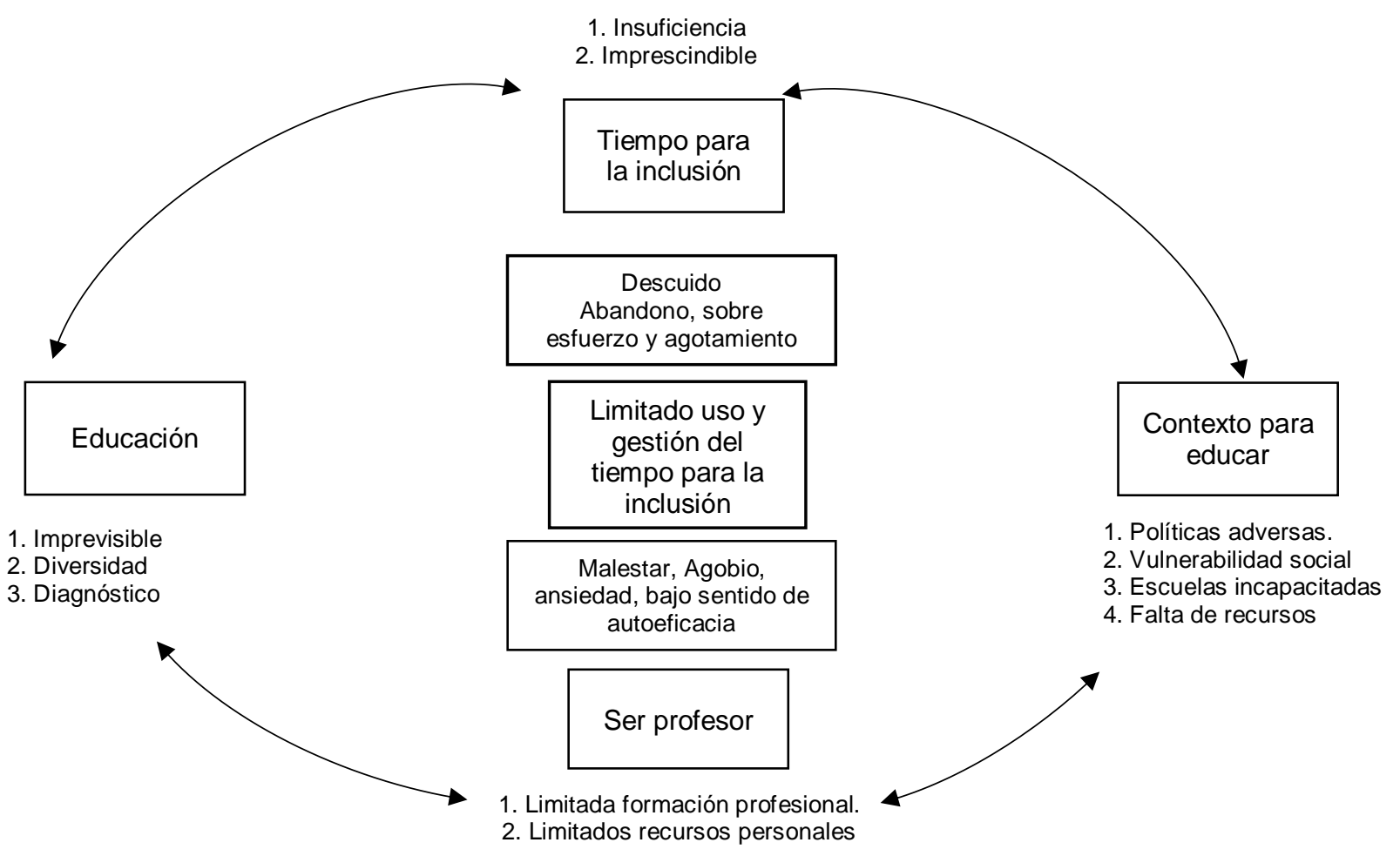

Fig. 5: Codificación selectiva. Modelo comprensivo de TS sobre el tiempo escolar y la inclusión educativa

\section{DISCUSIÓN}

Este trabajo tuvo como objetivo describir e interpretar las TS de los profesores, sobre el tiempo escolar y su relación con la inclusión educativa. En los contextos educativos, las TS ocupan un rol importante, porque permiten integrar la experiencia con un saber más abstracto y cercano al conocimiento, permitiendo a los profesores proporcionar explicaciones de los fenómenos educativos, orientando desde allí la toma de decisiones, las prácticas pedagógicas e incluso justificando la acción profesional (Koubek \& Janík, 2015). Estas TS emergen con más presencia en el ejercicio profesional, cuando el profesor no cuenta con teorías científicas desde donde abordar un fenómeno (Koubek \& Janík, 2015). En este estudio, lo anterior parece manifestarse para dos importantes temáticas que se encuentran omnipresentes en las escuelas y que han sido asociadas a la eficacia educativa: el tiempo escolar y la inclusión educativa. 
El primer objetivo de este trabajo, fue identificar la forma en que estos profesores le otorgan sentido y significado al tiempo escolar y a la inclusión educativa. Para este grupo de profesores, ambas variables son claves para el éxito educativo y requieren en el profesorado, un mayor desarrollo profesional por la escaza formación que consideran tener al respecto. Con respecto al tiempo escolar, ha sido conceptualizado como un fenómeno omnipresente en la profesión docente y en los contextos educativos (Ather et al., 2016; Rubio et al., 2019). Básicamente, los estudios muestran que los significados atribuidos a este recurso implican la valoración del tiempo como una oportunidad para la transformación educativa y la compensación de las desigualdades sociales con las que ingresan los estudiantes a las escuelas, lo que contrasta con la evaluación de escases del mismo (Eriksson et al., 2018; Wang, 2011). En cuanto a la inclusión educativa, en la literatura se la ha considerado como una forma de disminuir las desigualdades sociales que llevan a la exclusión y de hacer efectiva una educación de calidad para todos. Si bien hay evidencia de que los docentes tienen actitudes positivas hacia la inclusión escolar, son las competencias auto percibidas para esta tarea y los significados construidos al respecto los que requieren de un cambio mediante la educación formal (Aas, 2019; Knauf \& Graffe, 2016; Pick et al., 2016; Sharma \& Sokal, 2015). Estos resultados son coincidentes con lo encontrado en este trabajo, aunque cuando se intersecta el tiempo escolar con la inclusión educativa, las dificultades para instalar prácticas inclusivas se hacen aún mayores.

El segundo objetivo específico permitió comprender cómo los profesores explican su formación profesional, en relación al tiempo y la inclusión educativa. Básicamente, este grupo de profesores identifica dos fuentes de conocimiento: la formación inicial y la formación mediante el ejercicio profesional. El primer proceso formativo se califica como insuficiente para que los profesores logren dominio en el uso y gestión del tiempo para la inclusión del estudiantado, mientras que el segundo, es mejor valorado, siempre que el docente adquiera saberes en un contexto escolar inclusivo. La gestión y uso del tiempo es un problema presente en la profesión docente (Wang, 2011) y algunos estudios evidencian que los profesores no logran utilizar adecuadamente este recurso, por ejemplo, para la instrucción (Dougherty et al., 2019), siendo una de las causas la limitada formación al respecto. Para el caso de la inclusión educativa, los estudios muestran que se requiere de ambos procesos formativos: básicamente, las experiencias de capacitación, la introducción de programas de estudio sobre inclusión educativa y la formación continua, conjuntamente con experiencias profesionales de enseñanza a estudiantes con necesidades educativas especiales, son favorecedores del desarrollo de creencias positivas hacia la inclusión, de la adquisición de competencias al respecto y una mayor autoeficacia profesional (You et al., 2019). Sin embargo, al igual que los hallazgos encontrados, la evidencia actual muestra que la formación para la inclusión educativa es insuficiente, constituyéndose en un obstáculo para la formación de los estudiantes con necesidades educativas especiales (Mafra et al., 2018).

En el tercer objetivo, se buscó determinar los factores que promueven y obstaculizan la inclusión educativa, en función del tiempo escolar. Con respecto a los obstaculizadores, los profesores identifican principalmente factores considerados externos a su persona y sentidos con un menor grado de controlabilidad, señalando a las políticas y micropolíticas educativas como adversas para dedicar tiempo a la inclusión educativa. El factor socioeconómico también es identificado como un factor externo y de baja controlabilidad, que se asocia a una mayor vulnerabilidad en el estudiantado, cuando este es bajo. Como atribución interna, se identifica una débil formación profesional. Actualmente, en países desarrollados se ha avanzado en políticas favorecedoras de la inclusión educativa y una de las limitaciones al respecto es la evaluación del resultado de estas políticas, al interior de las escuelas (Anderson \& Boyle, 2015). En Latinoamérica, en cambio, algunas las brechas políticas a superar son (a) la identificación temprana de los estudiantes con necesidades educativas especiales; (b) la implementación de estándares universales de accesibilidad; (c) la asignación de más recursos a las escuelas; (d) la capacitación del profesorado y (e) la disponibilidad de ayudantes de aula capacitados (Hincapié et al., 2019). Los estudios de TS han evidenciado que cuando los profesores incorporan en la estructura argumentativa de sus explicaciones, causas o consecuencias de atribución externa, aumenta la sensación de descontrol, las emociones negativas y disminuye la agentividad del profesor (Koubek \& Janík, 2015). Desde los hallazgos de este estudio, esta sería una condición frecuente en el profesorado, lo que podría contribuir en la comprensión de las dificultades para la gestión y uso del tiempo escolar y la inclusión educativa. Otros estudios develan que los problemas para la implementación de la inclusión se relacionan con barreras físicas y actitudinales (Pappas et al., 2018), el nivel socioeconómico y cultural, el acceso físico al establecimiento, los recursos técnicos y humano y la infraestructura. Lo anterior invita a identificar con mayor precisión y desde otros diseños metodológicos, las características de estas condiciones y desafía a los operadores políticos a superar estas adversidades.

En cuanto a los facilitadores, este grupo docente señala la gestión del tiempo y de recursos desde la organización educativa, y la filosofía inclusiva de la escuela. Respecto del tiempo escolar, los estudios en centros educativos latinoamericanos evidencian que la extensión de la jornada escolar por sí sola no se asocia necesariamente a una mejora educativa, sino que más bien una adecuada gestión de este recurso es lo que podría impactar positivamente en los resultados educativos. Así, una adecuada gestión del tiempo posibilitaría habilitar nuevas formas de enseñanza y de aprendizaje para la diversidad estudiantil, en ámbitos organizativos- 
curriculares como facilitadores de la inclusión educativa (Rubio et al., 2019). Igual situación ocurriría con una eficiente administración de los recursos materiales para la enseñanza. El avance hacia la construcción de una cultura inclusiva podría ser la clave para mejorar la atención a la diversidad del estudiantado. Para Dwi Budiarti y Sugitox (2018), precisamente la instalación de una cultura inclusiva es la que da paso a la elaboración de una filosofía y política al respecto, lo que regula finalmente las prácticas para la inclusión. Sin embargo, a pesar de que en Latinoamérica se ha avanzado en la construcción de un marco legal y científico para la inclusión educativa, el problema parece ser justamente la generación de una cultura inclusiva al interior de los centros educativos (Hincapié et al., 2019).

Este estudio también permitió identificar propuestas de mejora de la gestión y uso del tiempo para la inclusión educativa. Destacaron dos TS, con atribución interna y externa y, por lo tanto, con mayor y menor sentido de autoeficacia, responsabilización por su implementación y agencia docente. La primera de éstas, identifica en el profesorado la necesidad de gestionar mejor el tiempo para la enseñanza y el aprendizaje y de lograr un mayor involucramiento en este proceso, factores que podrían considerarse como "una actitud de mayor compromiso docente". En cambio, la segunda TS posiciona en la escuela la necesidad mejorar la gestión del tiempo y de dotar de una mayor cantidad de asistentes y profesionales capacitados, para apoyar la labor del profesorado para la inclusión. Si bien estas propuestas podrían asociarse a mejoras en la gestión y uso del tiempo escolar y de la inclusión educativa, destaca la simplicidad de las TS del profesorado. Esto advierte de la necesidad de favorecer en el profesorado espacios de reflexión y análisis, para reconstruir TS más complejas (Knauf \& Graffe, 2016), que encadenen a las causas que reconocen como favorecedoras e inhibidoras del uso y gestión del tiempo para la inclusión educativa, con múltiples alternativas de solución, sobre todo, de atribución interna, viables, eficientes y que se asocien a una mayor autoeficacia y agentividad docente.

Finalmente, en este trabajo se propone un modelo comprensivo, que aporta en comprensión respecto de las TS docentes acerca de la gestión y uso del tiempo para la inclusión educativa y su relación con las prácticas pedagógicas. En este modelo, se puede entender el ejercicio profesional docente, en base a cuatro ejes que interrelacionadamente impactan en un adecuado o inadecuado uso y gestión del tiempo para la inclusión educativa: (a) el ser profesor, que implica cómo el docente se identifica con su profesión o se caracteriza como profesional y desde allí, piensa la gestión y uso del tiempo para la inclusión educativa; (b) la educación, a la cual se le otorga sentido desde asunciones ontológicas sobre el estudiantado y la enseñanza y el aprendizaje; (c) el contexto de trabajo docente, permeado por políticas, micropolíticas y medidas de presión por la calidad educativa, que tienden a no favorecer el uso y gestión del tiempo para la inclusión; y (d) el significado del tiempo escolar y su relación con la inclusión, que finalmente es considerado un recurso imprescindible, pero escaso, a partir de los tres ejes anteriores. Lo anterior, podría relacionarse con el frecuente agobio laboral del que advierten los profesores, con el abandono de la profesión (Chambers et al., 2019) y con limitaciones en las prácticas pedagógicas para favorecer la inclusión educativa, desde el tiempo escolar.

Este estudio tiene algunas limitaciones. En primer lugar, es necesario avanzar en estudios con diseños cuantitativos, que permitan acceder a muestran más amplias y diversas, que permitan complementar los resultados obtenidos. También queda como interrogante la perspectiva de otros actores educativos, que son clave para el éxito educativo, la gestión y uso del tiempo escolar y la inclusión educativa, como los directivos y los padres de los estudiantes. Los estudios acerca del tiempo escolar siguen siendo escasos (Dougherty et al., 2019) y mucho más la relación de éste recurso con la inclusión educativa. Aunque el tiempo es una temática de frecuente conversación en las instituciones educativas y presente en las políticas sobre la educación, su estudio sistemático requiere de mayores esfuerzos e iniciativas, en este caso, para promover desde allí una educación más justa, equitativa, de calidad e inclusiva.

\section{CONCLUSIONES}

En base a los resultados obtenidos en el estudio, se puede concluir: (a) los profesores reconocen la relevancia del tiempo y la inclusión, en el contexto educativo, sin embargo, advierten de una limitada formación al respecto; (b) planificar la inclusión educativa es una tarea difícil, dado que desde los profesores, la educación es un proceso imprevisible y la diversidad estudiantil es cada vez mayor, siendo el tiempo no lectivo un recurso insuficiente para favorecer la inclusión en las escuelas; (c) existen obstaculizadores y facilitadores de la inclusión educativa, siendo los primeros predominantes, lo que conlleva a sentimientos de baja eficacia del profesorado para lograr esta tarea; (d) las medidas de mejora sobre el uso del tiempo para la inclusión educativa, destacan factores personales del profesor y escolares: tanto los profesores como la institución educativa deben aprender a gestionar mejor el tiempo escolar, se requiere de una mayor dotación de asistentes de la educación que apoyen la labor docente y los profesores deben focalizarse mayormente en la enseñanza y aprendizaje del estudiante; (e) finalmente, la teoría comprensiva que explica el uso del tiempo escolar para la educación inclusiva, incluye asunciones ontológicas de lo que es educar, significados del tiempo y la inclusión educativa, caracterizaciones del contexto educativo y del ser profesor, que tienden a limitar la gestión del tiempo para la inclusión educativa. 


\section{AGRADECIMIENTOS}

Los autores agradecen al proyecto Fondecyt N 1180918 denominado "Teorías Subjetivas de Profesores(as) sobre el tiempo escolar: su variación según los años de experiencia y los contextos laborales", financiado por el Consejo Nacional de Ciencias y Tecnología de Chile (Conicyt).

\section{REFERENCIAS}

Aas, H.K., Teachers Talk on Student Needs: Exploring How Teacher Beliefs Challenge Inclusive Education in a Norwegian Context, doi: 10.1080/13603116.2019.1698065, International Journal of Inclusive Education, (2019)

Adams, R.V., y Blair, E., Impact of Time Management Behaviors on Undergraduate Engineering Students' Performance, doi: 10.1177/2158244018824506, SAGE Open, 9(1), 1-11 (2019)

Álvarez, D.R., y Lobo, G.P., El Tiempo, Uso y Abuso. Información Tecnológica, 31(2), http://dx.doi.org/10.4067/S071807642020000200073, 73-80 (2020)

Amor, A.M., Hagiwara, M. and five other authors, International Perspectives and Trends in Research on Inclusive Education: A Systematic Review, doi: 10.1080/13603116.2018.1445304, International Journal of Inclusive Education, 23(12), 1277-1295 (2019)

Anderson, J., y Boyle, C., Inclusive Education in Australia: Rhetoric, Reality and the Road Ahead, doi: 10.1111/14679604.12074, Support for Learning, 30(1), 4-21 (2015)

Ather, K.H., Khan, M.T., Khalil, A.A., y Faisal, I., Exploring Relationship of Time Management with Teachers' Performance, Bulletin of Education and Research, ISSN-0555-7747, 38(2), 249-263 (2016)

Berda, A., y Ochieng, J., Determining Influence of Teacher's Workload on Academic Performance in Secondary Schools, Suba Sub-County Kenya, doi:10.14738/assrj.63.6128, Advances in Social Sciences Research Journal, 6(3), 287-295 (2019)

Booth, T., y Ainscow, M., Index for Inclusion (3rd ed.), Bristol, Centre for Studies on Inclusive Education (2011)

Castro, P.J., González, I.N., y otros tres autores, Teachers' Subjective Theories about Time: Negative Emotional Meaning, Action Maintaining and Orientation to the Past, Psychology and Education. An Interdisciplinary Journal, 56(2), $1-29(2019)$

Catalán, J.R., Hacia la Formulación de una Teoría General de las Teorías Subjetivas, Psicoperspectivas, Individuo y Sociedad, 15(1), 53-65 (2016).

Chambers, J., Johnson, A., Jones-Rincon, A., Tsatenawa, V., y Howard, K., Why do teachers leave? A Comprehensive Occupational Health Study Evaluating Intent-to-quit in Public School Teachers, doi: 10.1111/jabr.12160, J. Appl. Behav. Res., 24(1), 1-13 (2019)

Demirkasimoglu, N., The Meaning of Work in Teachers' Lives: A Qualitative Study, doi: 10.1080/09720073.2015.11891894, Anthropologist, 22(2), 412-423 (2015)

Dougherty, C., Walsh, C., y Swan, K., Reimagining Time School for all Students, Unlocking Time, Education Commission of the States, Denver, CO (2019)

Dwi Budiarti, N., y Sugito, S., Creating Inclusive Culture of Elementary Schools: A Case Study in Karangmojo Subdistrict, Gunungkidul Regency, doi: 10.26811/peuradeun.v6i2.237, The International Journal of Social Sciences, 6(2), 307-324 (2018)

Eriksson, E., Björklund, L., y Thornberg, R., A Qualitative Study of Primary Teachers' Classroom Feedback Rationales, doi: 10.1080/00131881.2018.1451759, Educational Research, 60(2), 189-205 (2018)

Esteban-Guitar, M., Lalueza, J.L., Zhang-Yu, C., y Llopart, M., Sustaining Students' Cultures and Identities. A Qualitative Study Based on the Funds of Knowledge and Identity Approaches, doi: 10.3390/su11123400, Sustainability, 11(12), 1-12 (2019)

Flick, U., An introduction to Qualitative Research (sixth edition), Sage, Germany (2019)

Garzia, M., Mangione, G.R., Longo, L., y Chiara, M., Spaced Learning and Innovative Teaching: School Time, Pedagogy of Attention and Learning Awareness, doi: 10.1515/rem-2016-0004, REM - Research on Education and Media, 8(1), 1-16 (2016)

Hagiwara, M., Shogren, K.A., y otros seis autores, International Trends in Inclusive Education Intervention Research: $A$ Literature Review, Education and Training in Autism and Developmental Disabilities, 54(1), 3-17 (2019)

Hincapié, D., Duryea, S., y Hincapié, I., Education for All. Advancing Disability Inclusion in Latin America and the Caribbean, Inter-American Development Bank (2019)

Knauf, H., y Graffe, S., Alltagstheorien über Inklusion Inklusion aus Sicht Pädagogischer Fachkräfte in Kindertageseinrichtungen, doi: 10.1026/2191-9186/a000281, Frühe Bildung, 5(4), 187-197 (2016)

Koubek, P., y Janík, T., Výzkumy Subjektivních Teorii Učitelů v Kontextu Profesního Rozvoje: Přehledová Studie, doi: 10.5817/SP2015-3-4, Studia Paedagogica, 20(3), 47-68 (2015) 
Madani, R.A., Analysis of Educational Quality, a Goal of Education for All Policy, doi: 10.5539/hes.v9n1p100, Higher Education Studies, 9(1), 100-109 (2019)

Mafra, F.V., Matheus, E.G., Cezar, P., Lozano, D., y Gomes C., Training of University Professors and Their Meaning for Inclusive Education, doi: 10.1590/s1678-4634201844176672, Educação e Pesquisa, 44, 1-19 (2018)

Massouti, A., Rethinking Teacher Education for Inclusion: A literature Review, Canadian Journal for New Scholars in Education, 10(1), 160-168 (2019)

Moliner, O., Traver, J.A., Ruiz, M.A., y Segarra, T. Estrategias que Inciden en los Procesos de Democratización de la Escuela. Una Aproximación Teórica, Revista Electrónica de Investigación Educativa, 18(2), 116-129 (2016)

Pappas, M.A., Papoutsi, C., y Drigas, A.S., Policies, Practices, and Attitudes toward Inclusive Education: The Case of Greece, doi: 10.3390/socsci7060090, Soc. Sci. 7(6), 1-15 (2018)

Peng, Y., y otros nueve autores, Expanded In-School Instructional Time and the Advancement of Health Equity: A Community Guide Systematic Review, doi: 10.1097/PHH.0000000000000834, J Public Health Manag Pract., 25(6). 584589 (2019)

Pick, G., Holzinger, A., y Kopp-Sixt, S., The Special Education Teacher Between the Priorities of Inclusion and Specialization, doi: 10.1080/13603116.2015.1115559, International Journal of Inclusive Education, 20(8), 828-843 (2016)

Przibilla, B., Linderkamp, F., y Krämer, P., Subjektive Definitionen von Lehrkräften zu Inklusion - eine explorative Studie, URN: urn:nbn:de:0111-pedocs-165965, Empirische Sonderpädagogik, 10(3), 232-247 (2018)

Rubio, J., Cuadra, D.J., y otros tres autores, Tiempo Escolar: una Revisión Teórica sobre Estudios Empíricos Realizados en Centros Educativos Latinoamericanos, doi: 10.22201/iisue.24486167e.2019.164.59071, Perfiles Educativos, XII(164), 100-117 (2019)

Sharma, U., y Sokal, L., The Impact of a Teacher Education Course on Pre-service Teachers' Beliefs about Inclusion: An International Comparison, doi:10.1111/1471-3802.12043, Journal of Research in Special Educational Needs, 15(4), 276$284(2015)$

Strauss, A., y Corbin, J., Bases de la Investigación Cualitativa. Técnicas y Procedimientos para Desarrollar la Teoría Fundamentada, Editorial Universidad de Antioquia, Colombia (1998)

Taylor, S.J., y Bogdan, R., Introducción a los Métodos Cualitativos de Investigación, Paidos, Barcelona (1987)

Wang, D., The Dilemma of Time: Student-centered Teaching in the Rural Classroom in China, doi:10.1016/j.tate.2010.07.012, Teacher and Teaching Education, 27(1), 157-164 (2011)

You, S., Kim, E.K., y Shin, K., Teachers' Belief and Efficacy Toward Inclusive Education in Early Childhood Settings in Korea, doi: 10.3390/su11051489, Sustainability, 11(1489), 1-12 (2019) 
